\title{
MODELLING OF SIMPLY SUPPORTED CIRCULAR DIAPHRAGM FOR TOUCH MODE CAPACITIVE SENSORS
}

\author{
Sumit Kumar Jindal, Sanjeev Kumar Raghuwanshi \\ Department of Electronics Engineering, Indian School of Mines, Dhanbad, Jharkhand, India \\ e-mail: sumitjindal08@gmail.com
}

\begin{abstract}
This paper describes the power series solution for modelling of the simply supported circular diaphragm deflection under uniform load. The parameters such as touch point pressure and touch radius are defined. Moreover, these parameters are also computed by the algorithm proposed in the paper. Therefore, the power series solution can be applied for touch mode operation.
\end{abstract}

Keywords: touch mode, capacitive pressure sensor, circular diaphragm, power series

\section{Introduction}

Miniaturisation and integration of electronic devices have made far reaching technological revolutions. Integration of micromechanics on the same chip has resulted in Micro-Electro-Mechanical System (MEMS) that have added newer dimensions to conventional ICs. With the advent of micromachining technology, which is compatible with the conventional IC industry, MEMS have become dominant in many areas of applications. MEMS have main applications in sensors, actuators and smart structures. Commercially successful devices and systems using MEMS technology include many microsensors (e.g. pressure sensors, inertial sensors, chemical sensors, etc.), microactuators (e.g. microvalve, micropumps, microrelays, etc.) and Microsystems (e.g. for chemical analysis, biological analysis, etc.) One of the most common MEMS devices are pressure microsensors. Due to demand for lower cost and smaller dimensions, transition from mechanical to silicon type has occurred. The concept of touch mode capacitive pressure sensors has several advantages over normal mode sensors (Ding et al., 1990; Hejarzaribi et al., 2011; Ko, ; Ko and Mehregany, 1992; Ko et al., 1996; Rosegren et al., 1992). As the result of effort to improve linearity and overload ability, the touch mode sensor has been developed. It has the advantages such as good linearity, large operating pressure range, zero suppression possibility and large overload protection. The touch mode capacitive pressure sensor consist of at least one pressure sensitive capacitor. One electrode is formed on the stationary substrate of silicon, glass or other insulating materials; the other is a diaphragm made of silicon, poly-silicon, silicon carbide, etc. When a pressure is applied, the diaphragm deforms and changes the gap between the two electrodes, hence, the capacitance will change (Wang, 1997).

In response to the load applied, the core of the touch mode capacitive sensor design is to compute the diaphragm deflection behaviour, which is controlled by sensor structural parameters. Based on the theory of pure bending of a circular plate (Timoshenko, 1959), we can solve this problem in different ways based on different assumptions: small deflection model, large deflection model and finite element analysis. The small deflection model is a simple model that neglects the strain of the middle plane in the plate, therefore it can only be used for approximation, assuming that the deflection at centre is small compared to the diaphragm thickness. Finite element analysis provides the accurate results but it takes a long time to compute and post data process. So these two methods have not been taken into consideration. The paper 
describes the application of the large deflection model in the circular diaphragm touch mode sensor design with a simplified simulation. It can be used not only as an analytical solution but also as a design tool that includes numerical computation and linear analysis.
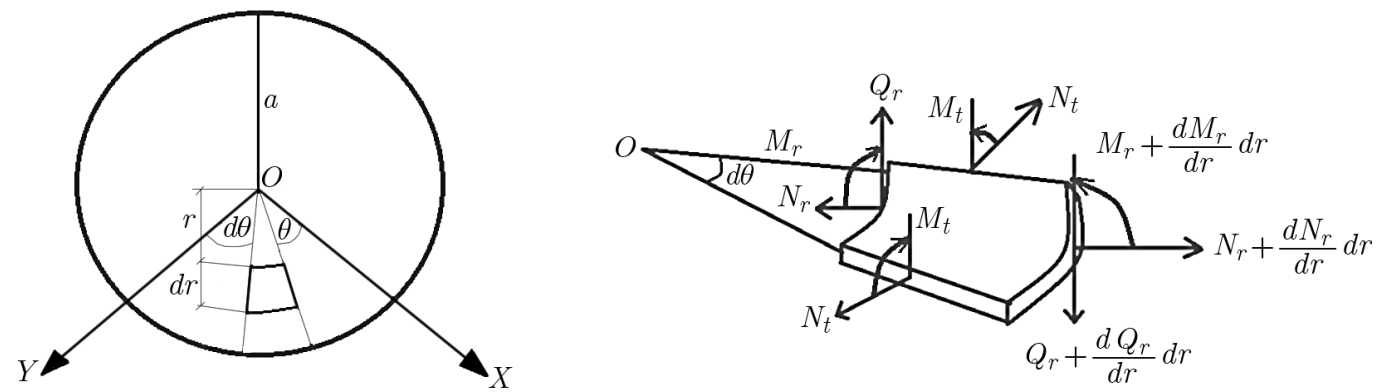

Fig. 1. Element of a circular plate for application in the touch mode capacitive sensor

We use a thin circular diaphragm to model the circular diaphragm as shown in Fig. 1, where $u$ stands for the displacement in the radial direction, $w$ is the deflection in the direction perpendicular to the plate, $E$ is the young's modulus, $\nu$ is Poisson's ratio, $h$ is thickness of the plate. The following parameters are listed below:

- strain in the radial direction

$$
\varepsilon_{r}=\frac{d u}{d r}+\frac{1}{2}\left(\frac{d w}{d r}\right)^{2}
$$

— strain in the tangential direction

$$
\varepsilon_{t}=\frac{u}{r}
$$

— tensile force per unit length in radial direction

$$
N_{r}=\frac{E h}{1-\nu^{2}}\left(\varepsilon_{r}+\nu \varepsilon_{t}\right)=\frac{E h}{1-\nu^{2}}\left[\frac{d u}{d r}+\frac{1}{2}\left(\frac{d w}{d r}\right)^{2}+\nu \frac{u}{r}\right]
$$

- corresponding tensile force per unit length in tangential direction

$$
N_{t}=\frac{E h}{1-\nu^{2}}\left(\varepsilon_{t}+\nu \varepsilon_{r}\right)=\frac{E h}{1-\nu^{2}}\left[\frac{u}{r}+\nu \frac{d u}{d r}+\frac{\nu}{2}\left(\frac{d w}{d r}\right)^{2}\right]
$$

— bending moment per unit length in radial direction

$$
M_{r}=-D\left(\frac{d^{2} w}{d r^{2}}+\frac{\nu}{r} \frac{d w}{d r}\right)
$$

— bending moment per unit length in tangential direction

$$
M_{t}=-D\left(\frac{1}{r} \frac{d w}{d r}+\nu \frac{d^{2} w}{d r^{2}}\right)
$$

where

$$
D=\frac{E h^{3}}{12\left(1-\nu^{2}\right)}
$$

— diaphragm stress

$$
\sigma_{r}=\frac{N_{r}}{h} \quad \sigma_{t}=\frac{N_{t}}{h}
$$


— bending stress

$$
\sigma_{r}^{\prime}=\frac{6 M_{r}}{h} \quad \sigma_{t}^{\prime}=\frac{6 M_{t}}{h}
$$

shearing force resultant from the lateral load

$$
Q_{r}=-N_{r} \frac{d w}{d r}-\frac{1}{r} \int_{0}^{r} q r d r
$$

Considering the equilibrium condition of the element of the circular plate as shown in Fig. 1, we get three governing equations by summing the forces, summing the moments and considering the relationship between the radial and tangential strain, respectively.

Finally, we have a set of governing equations as in the following

$$
\begin{aligned}
& r \frac{d N_{r}}{d r}+N_{r}-N_{t}=0 \\
& D\left(\frac{d^{3} w}{d r^{3}}+\frac{1}{r} \frac{d^{2} w}{d r^{2}}-\frac{1}{r^{2}} \frac{d w}{d r}\right)=N_{r} \frac{d w}{d r}+\frac{1}{r} \int_{0}^{r} q r d r \\
& r \frac{d}{d r}\left(N_{r}+N_{t}\right)+\frac{E h}{2}\left(\frac{d w}{d r}\right)^{2}=0
\end{aligned}
$$

This set of equations is universal to all situations of bending of the circular plate under load for non-touch operation. The equations can be modified for different shapes of load, such as uniform load, distributed load and concentrated load.

\section{Power series solution of the circular diaphragm under uniform load}

We have a set of non-linear differential equations governing the bending behaviour of the circular plate. Equations (1.1)where $N_{r}, N_{t}$ and $w$ are three unknown functions. Our task is to solve the equations to get an expression for $N_{r}(r), N_{t}(r)$ and $w(r)$.

First, we transform the equations into a dimensionless form by introducing the following notations

$$
p=\frac{q}{E} \quad S_{r}=\frac{N_{r}}{E h} \quad S_{t}=\frac{N_{t}}{E h}
$$

An exact solution to the problem can be obtained by a series method. Since the symmetry is axial, we have $d w / d r=0$ and $N_{r}=N_{t}$ at $r=0$. Since the radial couple must vanish on the edge, the further condition is

$$
\left[\frac{d}{d r}\left(\frac{d w}{d r}\right)+\frac{\nu}{r} \frac{d w}{d r}\right]_{r=a}=0
$$

Assuming the edge to be immovable, we have $S_{t}-\nu S_{r}=0$, which by Eq. $(1.1)_{1}$ is equivalent to

$$
\left[S_{r}(1-\nu)+r \frac{d S_{r}}{d r}\right]_{r=a}=0
$$

The function $S_{r}$ and $d w / d r$ may be represented again in form of the series

$$
\begin{aligned}
& S_{r}=\frac{h^{2}}{12\left(1-\nu^{2}\right) a^{2} \rho}\left(B_{1} \rho+B_{3} \rho^{3}+B_{5} \rho^{5}+\ldots\right) \\
& \frac{d w}{d r}=\frac{-h}{2 a \sqrt{3}}\left(C_{1} \rho+C_{3} \rho^{3}+C_{5} \rho^{5}+\ldots\right)
\end{aligned}
$$


where $\rho=r / a$. Using the series, we arrive at the following relations between constants $B$ and $C$

$$
\begin{aligned}
& B_{k}=-\frac{1-\nu^{2}}{2\left(k^{2}-1\right)} \sum_{m=1,3,5, \ldots}^{k-2} C_{m} C_{k-m-1} \quad k=3,5, \ldots \\
& C_{k}=\frac{1}{k^{2}-1} \sum_{m=1,3,5, \ldots}^{k-2} C_{m} B_{k-m-1}=8 C_{3}-B_{1} C_{1}+12 \sqrt{3}\left(1-\nu^{2}\right) \frac{p a^{4}}{h^{4}}=0 \quad k=5,7, \ldots
\end{aligned}
$$

Again, all constants can be easily expressed in terms of both constants $B_{1}$ and $C_{1}$, for which two additional relations, ensuring for boundary conditions, hold

$$
\sum_{k=1,3,5, \ldots} B_{k}(k-\nu)=0 \quad \sum_{k=1,3,5, \ldots} C_{k}(k+\nu)=0
$$

To start the resolution of the foregoing system of equations, suitable values of $B_{1}$ and $C_{1}$ may be taken on the basis of an approximate solution. Such a solution, satisfying equation (2.1), can be for instance of the form

$$
\frac{d w}{d r}=C\left(\beta \rho^{n}-\rho\right)
$$

where $C$ is the constant and $\beta=(1+\nu) /(n+\nu)(n=3,5, \ldots)$.

Further, we derive that

$$
S_{r}=c_{1}+\frac{c_{2}}{\rho^{2}}-\frac{C^{2}}{2}\left(\beta^{2} \frac{\rho^{2 n}}{n_{1}}-2 \beta \frac{\rho^{n+1}}{n_{2}}+\frac{\rho^{2}}{8}\right)
$$

Herein $c_{1}$ and $c_{2}$ are constants of integration and $n_{1}=4 n(n+1)$ and $n_{2}=(n+1)(n+3)$. The constant $C$ can be determined by some strain energy method.

Till here, the series solution is used for non-touched operation. Discontinuity is introduced when the plate touches the substrate. Therefore, the solution sets need to be modified for touch mode applications.

\section{Application to touch mode sensors}

Figure 2 shows the cross section of the touch mode capacitive sensor. Especially for touch mode application sensors of capacitive, we consider the problem with simply supported boundary conditions.

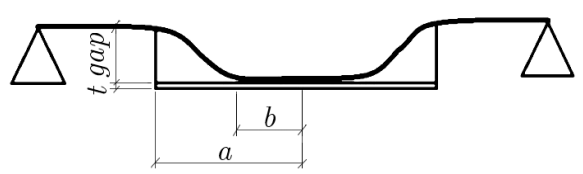

Fig. 2. Cross-section of the touch mode capacitive pressure sensor

As shown in Fig. 2, the distance between the bottom of cavity and the undeformed diaphragm is defined as gap. The pressure value at which the diaphragm deforms to touch the bottom of the cavity is defined as the touch point pressure. So the centre deflection of the diaphragm is equal to the gap. $a$ is radius of the diaphragm, $t$ is thickness of the insulating layer on the bottom of the cavity, $b$ is radius of the touched area that expands as the load increases, which is termed as 'touch radius'.

In the touch mode application, the touch radius $b$ is an important parameter. It is a function of the load $q$. On the introduction of touch radius and on the assumption that the untouched 
part of the diaphragm can be approximated by a diaphragm with reduced radius, we can rewrite the power series solution in the following form with $(\rho-b / h)$ as the variable

$$
\begin{aligned}
& S_{r}=c_{1}+\frac{c_{2}}{\rho^{2}}-\frac{C^{2}}{2}\left(\beta^{2} \frac{\rho^{2 n}}{n_{1}}-2 \beta \frac{\rho^{n+1}}{n_{2}}+\frac{\rho^{2}}{8}\right) \\
& S_{r}=c_{1}+\frac{c_{2}}{\rho^{2}}-\frac{C^{2}}{2}\left[\beta^{2} \frac{(\rho-b / h)^{2 n}}{n_{1}}-2 \beta \frac{(\rho-b / h)^{n+1}}{n_{2}}+\frac{(\rho-b / h)^{2}}{8}\right] \\
& \frac{w}{h}=\frac{1}{2 \sqrt{3}}\left(C_{0}+\frac{C_{1}}{2} \rho^{2}+\frac{C_{3}}{4} \rho^{4}+\frac{C_{5}}{6} \rho^{6}+\cdots\right) \\
& \frac{d w}{d r}=\frac{-h}{2 a \sqrt{3}}\left[C_{1}\left(\rho-\frac{b}{h}\right)+C_{3}\left(\rho-\frac{b}{h}\right)^{3}+C_{5}\left(\rho-\frac{b}{h}\right)^{5}+\cdots\right]
\end{aligned}
$$

Now the problem evolves into determination of the four constants $c_{1}, C_{0}, C_{1}$ and $b$. Practically all the four constants cannot be determined by iteration. To overcome this difficulty, we propose the following approach to simplify the computing procedure.

An approximation for calculation of the touch radius $b$ has been used. Before the touch point, the touch radius $b$ is certainly zero. For a given load $q$ larger than the touch point pressure, we can calculate the equivalent radius $a^{\prime}$ of a virtual circular diaphragm whose touch point pressure is $q$. This can be done by assigning the centre deflection the value of the gap (Timoshenko, 1959), as shown in Eq. $(3.2)_{4}$

$$
a^{\prime}=\sqrt[4]{\frac{g a p 64 D}{q}\left(1+0.488 \frac{g a p^{2}}{h^{2}}\right)}
$$

Now, the touch radius is the difference between the actual radius $a$ and equivalent radius $a^{\prime}$ as

$$
b=a-a^{\prime}
$$

$C_{0}$ should be decided by the maximum deflection of the circular diaphragm. Equations (3.4) and (3.5) shows the numerical evaluation of $C_{0}$ for both cases:

- before the touch point

$$
C_{0}=\frac{w_{\max }}{2 \sqrt{3} h}=\frac{1}{2 \sqrt{3} h} \frac{q a^{4}}{64 D}\left(1+0.488 \frac{w_{\max }^{2}}{h^{2}}\right)^{-1}
$$

- after the touch point

$$
C_{0}=\frac{w_{\max }}{2 \sqrt{3} h}=\frac{g a p}{2 \sqrt{3} h}
$$

Since $b$ and $C_{0}$ have been determined, the other two constants, $B_{0}$ and $C_{1}$ can be determined by iteration. Thus we can find a set of $B_{k}$ and $C_{k}$ to form the power-series solution.

\section{Results and discussions}

Based on the analysis in the previous Sections, a Matlab program has been written to obtain the characteristics. The program implements subroutines to compute the distribution of diaphragm deflection and stress under load. The characteristic of the capacitive sensor has also been calculated using the results for deflection.

The pressure versus capacitance plot has been obtained to describe the characteristics of the capacitive sensor as described by the equations analysed in the section above. 


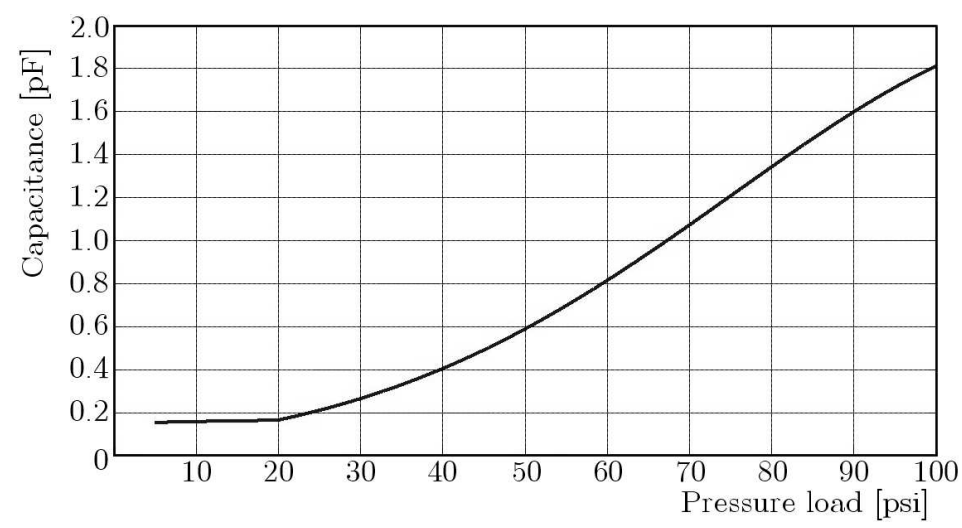

Fig. 3. Characteristics calculated by the power series solution

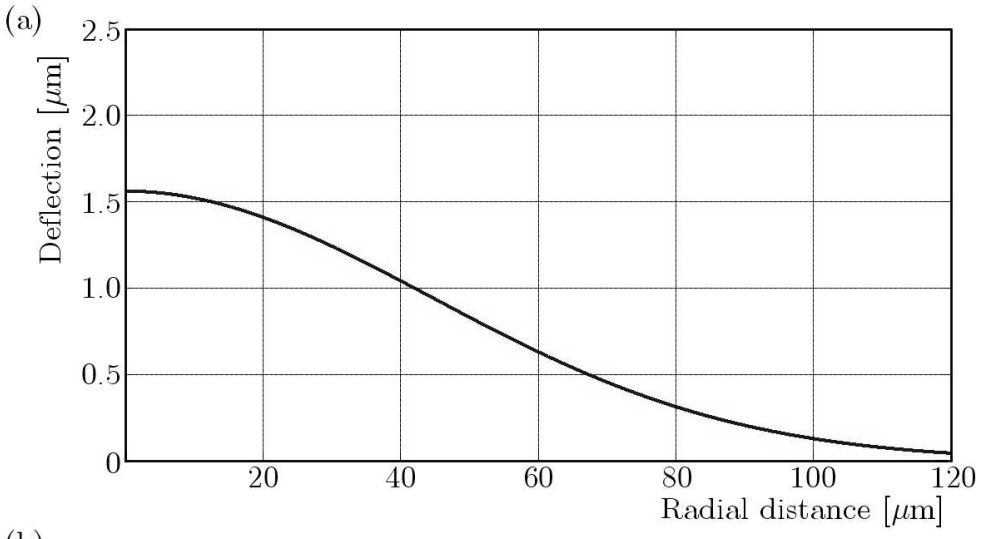

(b)

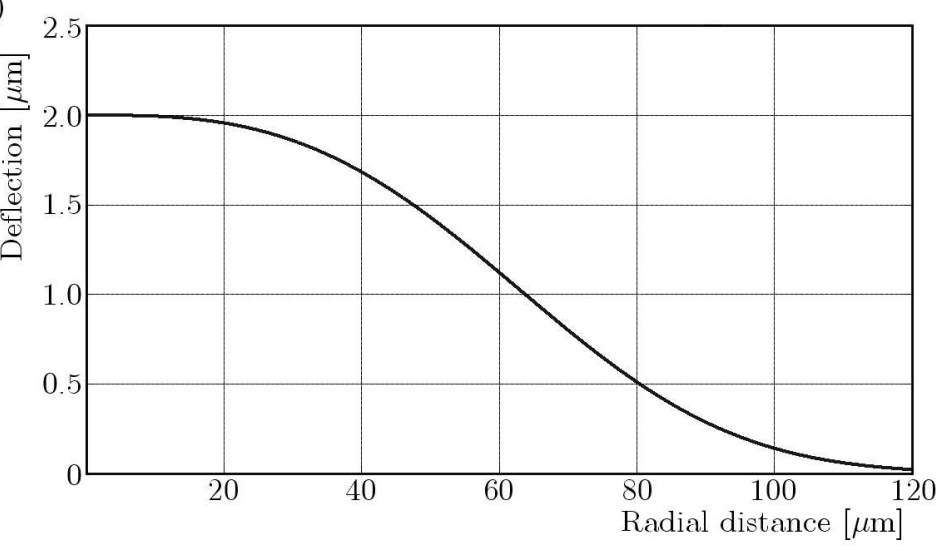

Fig. 4. Comparison of diaphragm deflection: (a) before the touch point, (b) after the touch point

Figure 4a shows the deflection behaviour of the diaphragm before the touch point when $q=$ $18 \mathrm{psi}(0.12 \mathrm{MPa}), a=100 \mu \mathrm{m}, h=2 \mu \mathrm{m}$, gap $=2 \mu \mathrm{m}$. In contrast, Fig. $4 \mathrm{~b}$ shows the deflection behaviour of the diaphragm after the touch point when $q=50 \mathrm{psi}(0.34 \mathrm{MPa}, a=100 \mu \mathrm{m}$, $h=2 \mu \mathrm{m}, g a p=2 \mu \mathrm{m}$. It is clear that deflection increases after the touch (Han and Shannon, 2009; Gupta and Ahmad, 2003; Lee and Jun, 2011).

Figure 5 shows the comparison of the diaphragm stress distribution under certain pressure load. Figure $5 \mathrm{a}$ is for 18 psi which is the before touch point while Fig. $5 \mathrm{~b}$ is after the touch point for a pressure of $50 \mathrm{psi}$. 

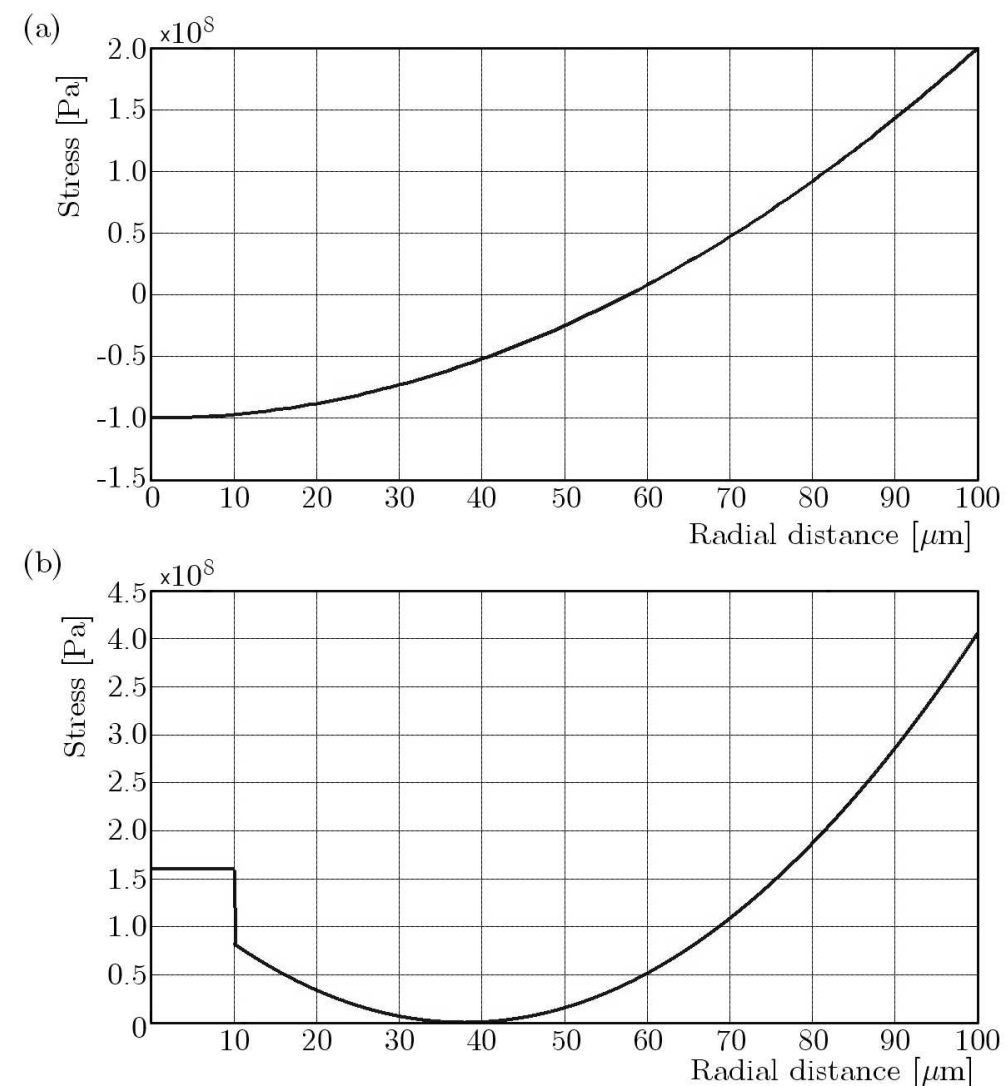

Fig. 5. Comparison of stress distribution: (a) before the touch point, (b) after the touch point

\section{Conclusion}

The power series solution is a promising method to do quick estimation of the diaphragm stress distribution and deflection. It can give relatively accurate results if they are compared with the results of ABAQUS (a finite element analysis software). For even quicker estimation, the analysis can be done using a spread sheet solution program by the help of Microsoft Excel and Visual Basic. Here, linear analysis is applied to the data within the operation range of sensors for a quick estimation. ABAQUS, on other hand, takes a large amount of time for data processing. It has been seen that the results of two differ by around $2-5 \%$, which is within an acceptable range. The deflection and stress on every node of the diaphragm is obtained from MATLAB, and they are used to calculate the performance of a touch mode capacitive pressure sensor. The touch point pressure is an important specification in the touch mode capacitive pressure sensor design. The linearity and sensitivity in the linear range are all related to the touch point pressure. The power series solution is specially useful for the touch mode sensor application because it treats the touch mode plate using the same form of the power series used for the untouched mode. Additionally, the power series solution is flexible. It can be easily implemented for the situation with other shapes of load provided the load can be expanded in a power series of radial displacement.

\section{References}

1. Ding X., Tong L., He W., Hsu J.T., Ko W.H., 1990, Touch mode silicon capacitive pressure sensor, ASME Winter Annual Meeting Dallas, 111-117

2. Gupta A., Singh R., Ahmad A., 2003, A capacitive pressure sensor for MEMS, Proceedings of SPIE, 5062, 450-454 
3. Han J.,Shannon M.A., 2009, Smooth contact capacitive pressure sensors in touch- and peelingmode operation, IEEE Sensor Journal, 9, 3, 199-206

4. Hejarzaribi Y., Hamidon M., Sidek R., Hossein K., 2011, Evaluation for Diaphragm's Deflection for touch mode MEMS pressure sensors, The International Arab Journal of Information Technology, 8, 2, 141-146

5. Ko W.H., 1996, Future of sensors and actuator systems, Sensors and Actuators A: Physical, 56, 193

6. Ko W.H., Mehregany M., 1992, Silicon microsensors and microactuators, Sensors and Materials, 3, 290-310

7. Ko W.H., WANG Q., WANG Y., 1996, Touch mode capacitive pressure sensors for industrial applications, Technical Digest Solid State Sensors and Actuators Workshop, Hilton Head Island

8. Lee M.K, Jun S.M., Kim S., Kim S., Choi B., 2011, Evaluation of sensitivity and linearity for touch-mode capacitive pressure sensor measuring the inner bladder pressure, International journal of Precision Engineering and Manufacturing, 112, 5, 907-912

9. Rosegren L., Soderkvist J., Smith L., 1992, Micromachined sensor structures with linear capacitive response, Sensors and Actuators A: Physical, 31, 200-205

10. Timoshenko S., 1959, Theory of Plates and Shells, The Maple Press, New York

11. WAng Q., 1997, Touch mode capacitive pressure sensor and interface circuits, PhD Dissertration in Dept. of EEAP, Cleveland, CWRU

Manuscript received February 21, 2014; accepted for print November 26, 2014 